\title{
HIGH FREQUENCY LATERAL TAPE MOTION AND THE DYNAMICS OF TAPE EDGE CONTACT
}

\author{
Ryan TAYLOR and Frank TALKE \\ Department of Mechnical and Aerospace Engineering, University of California at San Diego \\ UCSD/CMRR, 9500 Gilman Drive, La Jolla, CA, (858)-534-7578, USA \\ e-mail:taylor@talkelab.ucsd.edu
}

\begin{abstract}
Lateral motion of magnetic tape is measured on roller and porous air-bearing type tape drives. The lateral tape motion signal is high-pass filtered and the spectral components are analyzed. Time-frequency analysis is also performed. The effect of lateral motion of tape on tape pack winding is investigated and the frequencies observed during edge contact are analyzed. The effect of dynamic tension changes during high frequency lateral motion events is examined, and the tension difference across a section of tape is found to be correlated to the lateral motion of that section. LDV data of out-of-plane (transverse) tape motion is presented and its relationship to lateral tape motion and edge contact is discussed.
\end{abstract}

\section{INTRODUCTION}

In order to increase the track density of magnetic tapes, lateral motion above $1 \mathrm{kHz}$ must be reduced. Currently, the track width used in a typical magnetic tape drive is on the order of 26 um (768 tpi). For the track width to decrease to $1 \mathrm{um}$, the lateral tape motion above $1 \mathrm{kHz}$ must be reduced to less than $100 \mathrm{~nm}$ peak to peak. Lateral motion below $1 \mathrm{kHz}$ is generally followed by a closed loop track following servo system.

Lateral motion of magnetic tape has been measured optically and magnetically for some time [1]. Lateral motion of axially moving materials has also been modeled by Ono [2] and Shelton [3]. The phenomenon of edge buckling of guided webs was studied by Lakshmikumaran [4], and the quality of factory-slit magnetic tape edges was investigated by Topoleski [5]. Currently, storage capacity in magnetic tape is limited by track density. When the tape moves laterally, a track following servo head attempts to follow that motion. Current track following servo heads are bandwidth limited to less than $1 \mathrm{kHz}$. Thus, if the tape moves faster than $1 \mathrm{kHz}$, the tape moves away from the read head and errors occur. There are many sources of high frequency lateral tape motion, including edge contact. Under normal operating conditions, the tape is under tension, and lateral vibration at frequencies higher than 1 $\mathrm{kHz}$ can occur. The frequency response of a tape that contacts a tape path element may include lateral displacement frequencies in excess of $5 \mathrm{kHz}$. Edge contacts are important and must be better understood to improve tape path mechanics.

\section{EDGE CONTACT AND HIGH FREQUENCY LATERAL TAPE MOTION}

In Fig. 1(a) a porous air bearing guided tape drive is shown, and in Fig. 1(b), a roller guided tape drive. Tape edge displacement measurements were performed at the head positions of each of these drives.

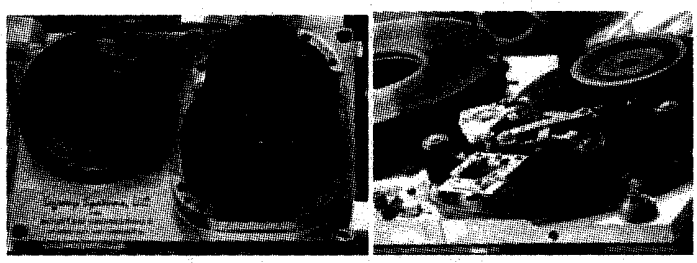

(a)

(b)

Fig. 1. (a) An externally pressurized porous air bearing guided tape drive, and (b) a commercial roller guided type tape drive

Typically, the supply and take-up reels on a tape drive produce a low frequency tape motion that dominates the lateral displacement of tape close to a reel. In general, the reels exhibit an axial run-out due to misalignment of the reel axis from the perpendicular direction. The fundamental frequency of this run-out displacement can be as low as $15 \mathrm{~Hz}$ for a full pack at $4 \mathrm{~m} / \mathrm{s}$, and as high as $160 \mathrm{~Hz}$ for a small pack at $10 \mathrm{~m} / \mathrm{s}$. In order to keep a constant tape speed, the rotational speed of the reel must be changed in a nonlinear fashion with time. This change in rotational speed affects the fundamental and, consequently, the harmonic frequencies of the reel run-out motion. This motion is transmitted to the tape through edge contact. A lateral force is exerted on the tape edge at least once every rotation from the reel flange run-out. Edge contact causes the tape to vibrate at frequencies much higher than the run-out frequency, and this is a source of high frequency lateral motion in tape.

In Figs. 2(a) and 3(a), lateral tape displacement is shown for a porous air bearing tape drive and a roller guided tape drive, respectively. The tape displacement is measured optically at the head position in each case. In Figs. 2(b) and 3(b), the lateral displacement signal is shown after high-pass filtering at $1 \mathrm{kHz}$.

We observe that bursts of high frequency motion exist in Figs. 2(b) and 3(b), occurring at times when the tape contacts a flange of a reel or a roller. In Fig. 2(b), the high frequency bursts occur at the times when the tape 
touches the bottom edge of the porous guide (there are no top flanges on these guides). In Fig. 3(b), the high frequency bursts occur at the top of the lateral excursions at exactly the interval of the reel runout edge contact. This indicates that the tape in Fig. 3(b) is contacting the top flange of the guide closest to the head. The high frequency motion appears to be caused by a series of delta function type impact forces as the tape edge contacts the inside of the reel flange.

(a)

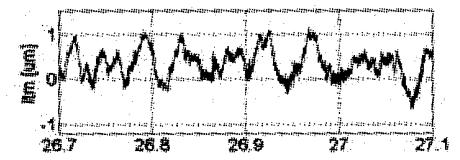

(b)

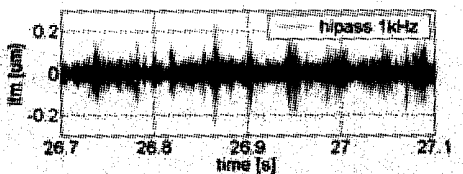

Fig. 2. Lateral tape displacement on an air bearing tape drive

(a)

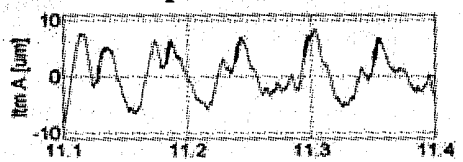

(b)

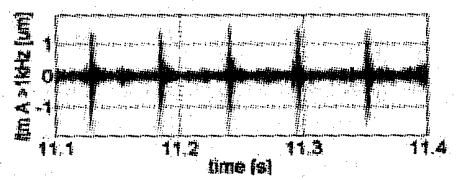

Fig. 3. Lateral tape displacement on a roller guided tape drive

In Fig. 4, a time-frequency analysis of the tape lateral displacement measurement for the air bearing drive is shown. We observe that the tape is being excited laterally in regular intervals similar to figures 2 and 3.

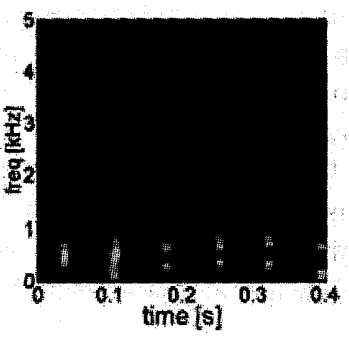

(a)

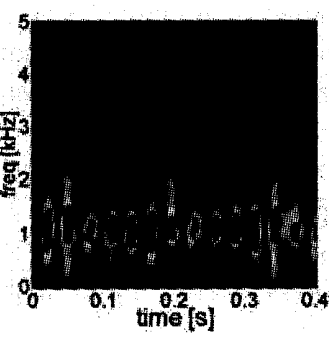

(b)
Fig. 4. Time-frequency plot of lateral tape displacement for the air bearing guided tape drive at (a) $4 \mathrm{~m} / \mathrm{s}$, and (b), $10 \mathrm{~m} / \mathrm{s}$.

Figure 4(a) shows that at $4 \mathrm{~m} / \mathrm{s}$, a frequency around $400 \mathrm{~Hz}$ is being excited, while at $10 \mathrm{~m} / \mathrm{s}$, a frequency around $1 \mathrm{kHz}$ is being excited (Fig. 4(b)). We conclude that the frequency of excitation is increasing with increasing tape speed.

\section{TENSION VARIATION AND HIGH FREQUENCY LATERAL TAPE MOTION}

Tape tension is an important parameter in the lateral motion of tape, since tension determines the overall stiffness of the system. In Fig. 5(a), the lateral tape displacement signal is shown for the air bearing guided tape drive, while Fig. 5(b) shows the tension variation before and after the head. The tension differential across the head is shown in Fig. 5(c), obtained by subtracting the tension signals in Fig. 5(b). We observe that the lateral tape displacement from Fig. 5(a) appears to be correlated with variation of the tape tension difference across the head (Fig. (5(c)) and the question arises whether the tension difference across the head is the cause or the result of lateral tape displacement. Further experiments will be needed to explore this phenomenon in more detail.

(a)

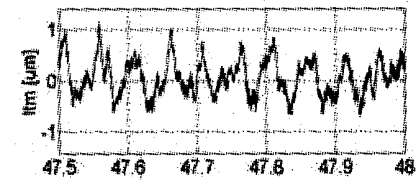

(b)

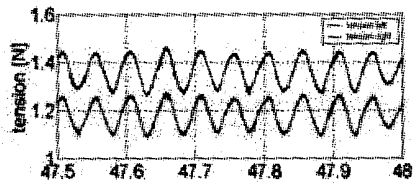

(c)

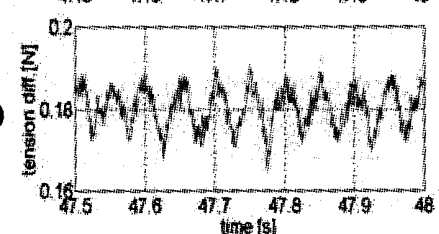

Fig. 5. Lateral tape displacement and tension variation on a porous air-bearing guided tape drive. (a) Lateral tape displacement, (b) tension variation on each side of the head, and (c) tension difference across the head.

\section{REFERENCES}

[1] Taylor R., Strahle P., Stahl J., and Talke F. (2000), "Measurement of Cross-Track Motion of Magnetic Tape", J. Information Storage and Processing Systems, Vol. 2, 255-262.

[2] Ono K., (1979), "Lateral Motion of an Axially Moving String on a Cylindrical Guide Surface", J. of Applied Mechanics, Vol. 46, 905-912.

[3] Shelton J., Reid K. (1971), "Lateral Dynamics of an Idealized Moving Web", ASME Journal of Dynamic, Systems, Measurement and Control, Vol. 93, 187-192

[4] Lakshmikumaran A., Wickert J. (1998), "Edge Buckling of Imperfectly Guided Webs", ASME Journal of Vibration and Acoustics, Vol. 120, 346-352

[5] Topoleski, J., and Bhushan, B. (2000), "Qualitative and Quantitative Evaluation of the Quality of Factory-Slit Magnetic Tape Edges", J. Information Storage and Processing Systems, Vol. 3, 267-275. 